\title{
El control intensivo de la presión arterial en pacientes sin diabetes con enfermedad renal crónica no reduciría la progresión de la enfermedad renal \\ Intensive blood pressure control in nondiabetic patients with chronic kidney disease would not prevent kidney
}

disease progression

\section{Objetivos}

Comparar la incidencia de eventos renales mayores en pacientes con enfermedad renal crónica (ERC) sin diabetes mellitus (DM) que recibieron un control intensivo de la presión arterial $(\mathrm{PA})$ vs el control estándar.

\section{Fuentes de datos}

Se realizó una búsqueda en las bases de datos PubMed, MEDLINE, Embase y The Cochrane Library, hasta marzo de 2016.

\section{Selección de estudios}

Se incluyeron ensayos clínicos aleatorizados (ECA), los cuales debían comparar una meta intensiva vs una estándar de PA en adultos con ERC sin DM, y reportar cambios en la tasa de filtrado glomerular (TFG), duplicación del nivel de creatinina, reducción en un $50 \%$ de la TFG, progresión a enfermedad renal terminal o mortalidad por cualquier causa.

\section{Extracción de datos}

Dos investigadores extrajeron los datos y evaluaron la calidad metodológica en forma independiente, resolviendo las diferencias a través de la discusión. La magnitud del efecto de los re- sultados finales continuos se expresan como diferencia media con su intervalo de confianza del $95 \%$ (IC 95\%) y los puntos finales dicotómicos, como riesgos relativos (RR) con su IC 95\%. Se utilizó un modelo de meta-análisis de efectos aleatorios para agrupar las medidas de efecto. Se realizaron análisis de metaregresión y de subgrupos para explorar heterogeneidad y se realizó un análisis de sensibilidad excluyendo a los estudios con datos faltantes y a aquellos que habían incluido algunos pacientes diabéticos, para evaluar la robustez de los hallazgos.

\section{Resultados principales}

Se incluyeron 9 ECA con un total de 8.127 pacientes y una mediana de seguimiento de 3,3 años. En comparación con el control estándar de la PA, el control intensivo no mostró diferencias significativas en ninguno de los resultados principales (ver Tabla 1). Los pacientes no pertenecientes a la etnia negra y aquellos con niveles elevados de proteinuria mostraron una tendencia a menor progresión de la enfermedad renal con un control más intensivo de la PA. Los resultados no variaron en el análisis de sensibilidad. La incidencia de eventos adversos no fue diferente entre ambos grupos, excepto para los mareos, que fueron algo más frecuentes en el grupo de control intensivo.

Tabla 1. Comparación del control intensivo vs estándar de la presión arterial en pacientes con enfermedad renal crónica sin diabetes.

\begin{tabular}{l|c}
\multicolumn{1}{|c|}{ Desenlaces } & Diferencia media (IC95\%) \\
\hline Cambio anual de la TFG (mL/min/1,73 $\mathbf{~ m}^{2} /$ año) & $0,07(-0,16$ a 0,29$)$ \\
\hline \multicolumn{1}{|c|}{ Desenlaces } & RR (IC95\%) \\
\hline Duplicación del nivel de creatininemia o reducción en un $\mathbf{5 0 \%}$ de la TFG & $0,99(0,76$ a 1,29$)$ \\
\hline Enfermedad renal terminal & $0,96(0,78$ a 1,18$)$ \\
\hline Mortalidad por cualquier causa & $0,95(0,66$ a 1,37$)$ \\
\hline
\end{tabular}

IC: intervalo de confianza; RR: riesgo relativo; TFG: tasa de filtrado glomerular.

\section{Conclusiones}

Una meta de PA por debajo del estándar actual no mostró beneficio adicional en cuanto a eventos renales durante un seguimiento de 3,3 años en pacientes con ERC sin DM. Sin embargo, los pacientes no pertenecientes a la etnia negra o aquellos con altos niveles de proteinuria podrían beneficiarse del descenso inten- sivo de la PA, con un riesgo de eventos adversos similar.

Fuente de financiamiento/conflicto de interés de los autores: National Health Research Institutes, Taiwan (NHRI-EX105-10510PC) y el Far Eastern Memorial Hospital, New Taipei City, Taiwan (FEMH-EX10510510PC). Los autores declaran que no existieron conflictos de interés.

\section{Comentario}

El meta-análisis aquí comentado plantea un interrogante de mucha relevancia para la comunidad médica: ¿Es el tratamiento intensivo de la PA mejor que el estándar en términos de eventos renales mayores y mortalidad en pacientes sin DM con ERC? Los estudios realizados a la fecha muestran resultados controvertidos, lo que se refleja en las referencias citadas en diversas guías, que recomiendan diferentes metas de PA para esta población, desde $<130 / 80$ hasta $<140 / 90 \mathrm{mmHg} .^{1-3} \mathrm{Si}$ bien este trabajo no mostró globalmente diferencias significativas, sí se observó una tendencia al beneficio de un tratamiento más intensivo de la PA en aquellos pacientes con proteinuria y no pertenecientes a la raza negra. El hecho de que los resultados no variaron al realizar un análisis de sensibilidad excluyendo a los estudios con datos faltantes y con pacientes con DM constituye una fortaleza del estudio. Sin embargo, deben tenerse en cuenta algunas cuestiones al interpretar este meta-análisis: primero, las técnicas utilizadas para medir la PA no fueron uniformes, variando desde el clásico método auscultatorio hasta las mediciones automatizadas no observadas, por lo que los niveles de presión obtenidos no son equiparables. Segundo, los parámetros utilizados para medir la intensidad del tratamiento son disímiles: algunos estudios eligieron la PA sistólica (PAS), otros la diastólica (PAD), y otros la presión arterial media (PAM). Más aún, entre los estudios que eligieron un mismo parámetro, las metas consideradas como "intensivas" fueron muy heterogéneas. Por ejemplo, el estudio JATOS ${ }^{4}$ consideró intensiva una meta de PAS $<140 \mathrm{mmHg}$, mientras que el SPRINT, ${ }^{5}$ una meta $<120$ $\mathrm{mmHg}$. Para profundizar esta dificultad, en muchos de los estudios no se alcanzó la meta considerada intensiva. Finalmente, aún en pacientes con proteinuria y que no son de raza negra, en los que podría ser beneficiosa una meta intensiva, no sabríamos, en base a este meta-análisis, qué parámetro utilizar (¿PAS? ¿PAD? ¿PAM?) y a qué objetivo apuntar ( $¿<120$ ?, ¿<140?).

\section{Conclusiones del comentador}

Si bien este estudio no parece sustentar un tratamiento más intensivo de la hipertensión en pacientes con enfermedad renal crónica, es fundamental evaluar al paciente individualmente. Los resultados aquí planteados no permiten extraer conclusiones con aplicación directa a la práctica clínica. 
Jessica Barochiner [ Sección Hipertensión Arterial, Servicio de Clínica Médica, Hospital Italiano de Buenos Aires. jessica.barochiner@hospitalitaliano. org.ar]

Barochiner J. El control intensivo de la presión arterial en pacientes sin diabetes con enfermedad renal crónica no reduciría la progresión de la enfermedad renal. Evid Actual Pract Ambul. 2018;21(4):106-107. Comentado de: Tsai WC y col. Association of Intensive Blood Pressure Control and Kidney Disease Progression in Nondiabetic Patients With Chronic Kidney Disease: A Systematic Review and Meta-analysis. JAMA Intern Med. 2017;177(6):792799. PMID: 28288249

\section{Referencias:}

1. Whelton PK y col. 2017ACC/AHA/AAPA/ABC/ACPM/AGS/APhA/ASH/ASPC/NMA/PCNA Guideline for the Prevention, Detection, Evaluation, and Management of High Blood Pressure in Adults: Executive Summary: A Report of the American College of Cardiology/American Heart Association Task Force on Clinical Practice Guidelines. J Am Coll Cardiol. 2017. pii: S0735-1097(17)41518-X.

2. James PA y col. 2014 evidence-based guideline for the management of high blood pressure in adults: report from the panel members appointed to the Eighth Joint National Committee (JNC 8). JAMA. 2014;311(5):507-20.

3. Mancia G y col. Task Force for the Management of Arterial Hypertension of the European Society of Hypertension and the European Society of Cardiology. 2013 ESH/ESC Practice Guidelines for the Management of Arterial Hypertension. Blood Press. 2014;23(1):3-16.

4. Hayashi K y col; JATOS Study Group. Impact of renal function on cardiovascular events in elderly hypertensive patients treated with efonidipine. Hypertens Res. 2010 Nov;33(11):1211-20.

5. SPRINT Research Group, Wright JT Jr y col. A Randomized Trial of Intensive versus Standard Blood-Pressure Control. N Engl J Med. 2015;373(22):2103-16.

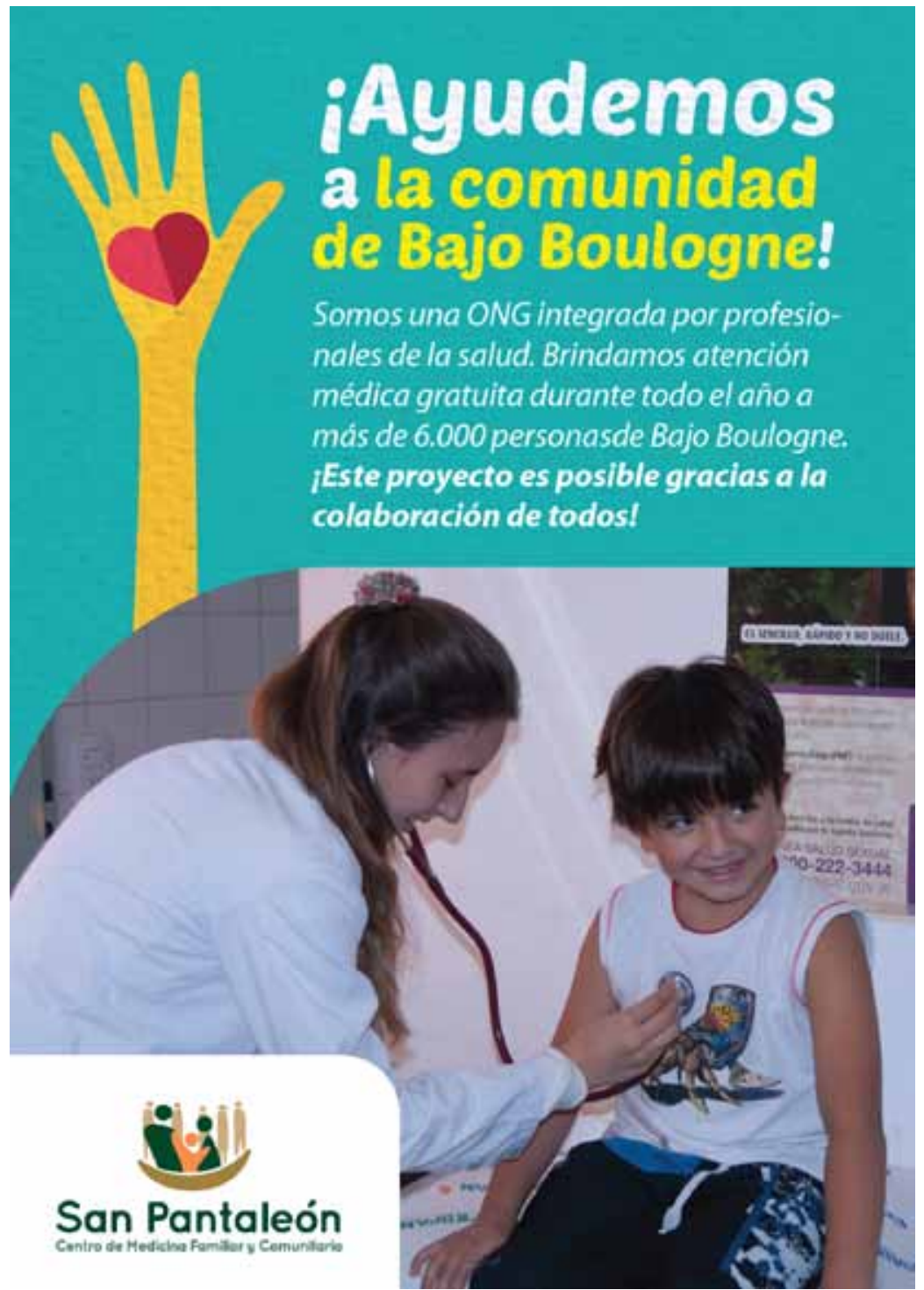

